\title{
Die Hohen Schulen und die Gesellschaft im 12. Jahrhundert
}

Jede höhere Kultur *) bringt die ihr gemäßen Formen höherer Bildung und höherer Schulen hervor. Die spezifisch europäische Form der hohen Schule, die Universität, ist eine Schöpfung des Hochmittelalters, genauer gesagt des 12. Jahrhunderts. In Bologna und Paris ist damals die Universität entstanden, und noch vor 1200 formt sich Oxford nach dem Vorbild von Paris. Kreuzzüge und Rittertum, höfische Kultur und Aufstieg der städtischen Bürgerfreiheit, Ausbreitung der volkssprachlichen Poesien und hochromanische und frühgotische Kunst sind die bekannten Kennzeichen dieses so unglaublich schöpferischen Zeitalters. Zugleich entsteht eine lateinische wissenschaftliche und theologische Literatur von solcher Vielfalt, daß kaum ein Gedanke der folgenden Jahrhunderte nicht schon hier in nuce vorweggenommen wurde, und von solchen Ausmaßen, da $B$ bis heute ein wesentlicher Teil ungedruckt ist, obwohl die Erforschung dieser sogenannten Frühscholastik heute eine Spezialwissenschaft bildet.

Universitas und studium generale heiBen die Namen, die die hohen Schulen in ihrer ausgebildeten Form seit dem 13. Jahrhundert führen. Beide Ausdrücke bezeichnen dieselbe Sache unter verschiedenen Aspekten - und der Historiker muß daran erinnern, daß sie nichts mit dem zu tun haben, was man heute oft in sie hineinlegt. Studium heiBt die wissenschaftliche Lehre, und ein Generalstudium ist ursprünglich ein allgemeines Studium, wo jedermann, gleich welches Standes oder welcher Herkunft, studieren kann und wo man schlieBlich die licentia ubique docendi erwirbt, so wie ein Generalkonzil ein allgemeines Konzil ist - den Gegensalz würde ein studium provinciale, eine Landes- oder Diözesanschule, bilden. Universitas nennt man die Körperschaft, die das Studium trägt - universitates, d. h. Körperschaften oder Genossenschaften, heiBen im kirchlichen und weltlichen Recht des Mittelalters Personenverbände der verschiedensten Art, von der Gesamtkirche, als Personenverband verstanden, über den Klerus einer Diözese bis zu den Bürgern einer Stadt, den Batern eines Dorfes oder den Meistern einer Zunft. An den Schulen bilden sich die zwei bekannten Typen: Bologna und die nach Bologneser Art verfaßten Hochschulen werden von einer universitas scholarium gebildet, in der allein die Studenten Glieder der Körperschaft sind. Die zu einem Collegium vereinten Doctores schwören dem studentischen Rektor einen Gehorsamseid, und dieser übt über sie eine äuBerst scharfe Disziplinargewalt mit Hilfe der denunciatores doctorum, einer Art studentischer Polizei. Paris dagegen und

*) Antrittsvorlesung, gehalten vor der Philosophischen Abteilung der Justus Liebig-Universität am 4. Februar 1964. Eine erweiterte und mit ausführlichen Quellen- und Literaturnachweisen versehene Fassung dieses Versuchs soll im Archiv für Kulturgeschichte erscheinen. 
die nach Pariser Muster verfaßten Hochschulen werden von der aniversitas magistrorum et scholarium getragen, der Genossenschaft der Lehrenden und Studierenden, die in ihrem Ursprung und Kern eine Genossenschaft der Magister ist.

Beides, die genossenschaftliche Verfassung der Schule wie ihre an keine Grenzen des Landes, des Standes, der Diözese oder des Ordens gebundene Wirksamkeit, ist dem früheren Mittelalter durchaus fremd. Bis ins späte 11. Jahrhundert hatte es in Europa nur Kílosterund Domschulen gegeben, die in der Regel rein interne Schulen für die Glieder des eigenen Konventes waren, und selbst wenn sie, wie einzelne Domschulen, auch auswärtige Kleriker ausbildeten, so jedenfalls unter der unmiltelbaren Aufsicht und Disziplin des dem Domkapitel angehörenden Domscholasters, der in Frankreich oft mit dem bischöflichen Kanzler identisch war. Seit dem Ausgang des 11. Jahrhunderts blühen plötzlich an zahlreichen Orten Frankreichs in großer Zahl Schulen neu auf, und die alten bei Domen und Klöstern entfalten eine große Regsamkeit. Dabei ist of genug unklar, wer die der Autorität des kirchlichen Lehramles zukommende Legitimation für die Lehrtätigkeit gibt und sie überwacht. Nennen wir nur das bekannteste Beispiel, die Lebensgeschichte PETER ABaElards. Dieser hört an verschiedenen Orten Frankreichs Vorlesungen über Dialektik, bis er zu dem berühmtesten Meister der Zeit, dem Pariser Archidiakon Wilhelm von Champeaux, gelangt. Nach kurzer Zeit entläuft er dem Lehrer, um diesem selbst Konkurrenz zu machen, und wo immer ABAELARD nun auftaucht, da schart sich eine Menge von Scholaren um ihn. Zuerst in Melun, dann in Corbeil - dann, nach neuen Studien bei Wilhelm von Champeaux, in Paris selbst. von dort vertrieben wieder in Melun, zurück zum Genoveva-Berg vor den Toren von Paris (nahe dem heutigen Pantheon), von wo aus seine Schule, wie er selbst sagt, die des Domschulmeisters auf der Ile de la Cilé belagert, während Wilhelm von Champeaux im Kloster Sankt Viktor, keine Viertelstunde Wegs von den beiden andern entfernt, eine öffentliche Schule als Konkurrenz unterhält. AbaELaRu gibt diese Schule auf, um in Laon Theologie zu studieren, und schließlich kann er auf der Ile de la Cité in Paris selbst lehren. Hier, auf dem ersten Höhepunkt seines Ruhmes, stürzt ihn der Privatunterricht, den er dem jungen Mädchen Héloise erteilt - so unvoreingenommen ist man schon, daß auch eine Frau den höchsten Unterricht genießen soll -, in die Katastrophe. Mönch geworden, lehrt ABAELARD in Saint-Denis - und plötzlich ist die alte Königsabtei von Scholarenhaufen übersät. Nach der Verurteilung zu Soissons und dem Konflikt mit den Mönchen baut er an einsamer Stätte in der Champagne bei Troyes sein Parakletkloster, um das herum alsbald eine Studentenstadt aufwächst; und als er nach vielen Jahren nach Paris zurückkehrt, erleben die Studien dort einen neuen Auftrieb.

Abaelaros Laufbahn ist das anschaulichste, aber gewiß nicht einzige Zeugnis dafür, daß in der ersten Hälfte des 12. Jahrhunderts Lehrer und Schüler sich den Platz ihrer Wirksamkeit suchen, daß 
Schulen mit dem Auftreten eines gefeierten Meisters aufblühen und vergehen. Wie aber sind die beständigen Universitäten entstanden?

In einer bedeutenden, an Material und Gedanken gleich reichen Studie ist Herbert Grundmann vor einigen Jahren zu dem Ergebnis gekommen, „primär und konstitutiv ... für Ursprung und Wesen der Universitäten als ganz neuartiger Gemeinschaftsbildungen" seien "weder die Bedürfnisse der Berufsausbildung noch staatliche, kirchliche oder sozialökonomische Impulse oder Motive, sondern kurz gesagt das gelehrte, wissenschaftliche Interesse, das Wissen- und Erkennenwollen" gewesen. Einer seiner Kritiker, WOLFram voN DEN Steinen, hat dazu bemerkt, dies ,klinge recht ideologisch“, und er hat gegenüber der Feststellung, die Universitäten seien um 1200 „ohne bewußtes Vorbild spontan aus Wissensdrang entstanden“, auf die enge Wechselbeziehung von wissenschaftlichem und praktischberuflichem Interesse, insbesondere bei den Juristen, aber auch bei den 'Theologen, hingewiesen ${ }^{1}$ ).

Mich dünkt, hier sind zwei Dinge zu unterscheiden: Ohne den von GRUNDMANN charakterisierten reinen Wissensdrang wäre gewiB keine Universität entstanden, und auch vON DEN STEINEN wird der letzte sein, der dies bestreitet. Aber der Geist allein vermag sich den Leib nicht zu schaffen.

Der christlichen Ethik war die curiositas, die Wißbegierde, stets suspekt erschienen, und die Autorität Augustins hatte dem Nachdruck verliehen. Nun aber lehrte selbst Hugo von St. VIkTor, der größte streng klösterliche Meister des Jahrhunderts, der wie kein anderer reines Leben, echte Forschung und gottesfürchtige Mystik so zu vereinen wubte, daß nicht einmal die Kollegen ihn zu schmähen wagten - Hugo, sage ich, lehrte: „Lerne alles, später wirst Du sehen, daß nichts überflüssig ist. Beschränkte Wissenschaft ist nicht erfreulich." War die Wissenschaft bisher in der Regel nur als Mittel zur Gotterkenntnis ethisch gerechtfertigt und nur von Einzelgängern um ihrer selbst willen geschätzt worden, so erhält sie nun in weiten Kreisen einen Eigenwert, und dieser geistige Wandel ist gewiß die erste Voraussetzung für das Werden der Universitäten.

Aber nur in Bologna und Paris kam es zu den neuen Gemeinschaftsbildungen, während doch das Streben zur Wissenschaft allgemein verbreitet war. Wäre GRUNDMANNs These ganz richtig, so müßte man staunen, daß es am Ende des 12. Jahrhunderts nur zwei Universitäten gibt, während doch an so vielen Plätzen Schulen entstanden waren. Bis zur Mitte des 12. Jahrhunderts und zum Teil darüber hinaus sind erst Laon, dann Reims, aber auch Tours, Orléans, selbst das kleine Melun, vor allem aber Chartres - alle nur 4-5 Tagereisen von Paris entfernt in der Francia gelegen - Plätze, die sich neben Paris durchaus sehen lassen können. Die Leistungen

1) Herbert Grundmann, Vom Ursprung der Universität im Mittelalter. Berichte über die Verhandlungen der Sächsischen Akademie der Wissenschaften, Phil.-hist. Klasse 103, 2, 1957. Zweite, mit einem Nachtrag versehene Auflage. Darmstadt, Wiss. Buchgesellschaft, 1960. Rezension von WOLFRAM vON DEN STEINEN, Historische Zeitschrift 186 (1958), S. 116-118. 
der Schule von Chartres, die den Platonismus belebt, aber auch bei der Rezeption des Aristoteles an der Spitze steht, die vor allem wie keine Schule sonst die auclores des Altertums liest und deren Sprache pflegt - sie stehen an geistigem Rang bis um 1150 gewiB nicht hinter dem zurück, was Paris hervorbringt. Hier in Chartres, wo wir noch heute mit Staunen am eben um 1150 geschaffenen Königsportal der Kathedrale Cicero und Aristoteles, Euklid und Pythagoras als Patrone der freien Künste einen Kranz un die Gottesmutter bilden sehen, hier, wenn überhaupt irgendwo, war der reine Wissensdrang zu Hause. Warum ist nur Paris, nicht auch Chartres, Universität geworden?

BERNHARD, der Kanzler von Charlres zwischen 1119 und 1124, hat in schönen Versen den Schlüssel zur Weisheit beschrieben:

mens humilis, studium quaerendi, vita quieta, scrutiniam tacitum, paupertas, terra aliena:

haec reserare solent multis obscura legendo.

(Demut im Sinn und eifriges Forschen und ruhiges Leben, Schweigsam und zäh untersuchen und arm sein, weit in der Fremde,

Vielen pflegt dies zu erschließen, was unbekannt war, durch Studieren.)

Hier sind die siltlichen Forderungen ihrem Wesen nach verbunden mit den - gleichfalls sitllich gewerteten --.- sozialen Gegebenheiten: paupertas, terra aliena. In der Fremde sucht der Student die Quelle der Wissenschaft, so wie der Ritter der Kreuzzugszeit sich in der Fremde bewährt; die frühere Bindung an die Diözese oder das Kloster wird nicht mehr nur von einzelnen, sondern ganz allgemein aufgegeben; sie soll aufgegeben werden, um den Studenten frei für die Wissenschaft zu machen. Armut ist, so will es scheinen, zugleich Folge des Lebens in der Fremde und Voraussetzung für die Freiheit zur Wissenschaft. Aber schon Hugo von SankT VikTok schrieb um 1135 in seinem Kommentar zu diesen Versen: „Was werden die Studenten unserer Zeit - man muB hinzufïgen: in Paris - dazu sagen können, die bei ihrem Studium nicht nur Entbehrung verachten, sondern sogar bemüht sind, noch reicher zu erscheinen, als sie sind? Man prahlt nicht mit dem, was man gelernt, sondern mit dem, was man ausgegeben hat!" Und Berniaros Nachfolger in Chartres, Gilbert Porketa, sah sich um dieselbe Zeil veranlaBı, diejenigen, die nicht Weisheit, sondern rasch erlernbare Wissensrezepte für den Erfolg im Leben suchten, auf die Vorzüge des Bäckerhandwerks hinzuweisen.

Es scheint, daß neben den rein geistigen auch ganz andere Kräfte und Molive mitgewirkt haben, die erst die Universitä als nene soziale Form möglich machten. ABAELARD selbst gesteht in seiner Autobiographie, er habe bis zu der groben Kalastrophe seines Lebens nur um Ruhmes und Geldes willen gelehrt. Das ist gewiß nicht naves Eingeständnis, sondern bewubte Selbststilisierung in dem literarischen 
Kunstwerk der historia calamitatum. Aber die Zeugnisse von ihm selbst und vielen kritischen Zeitgenossen bestätigen es: er wurde reich und berühmt. Dem entspricht es, daß er wie andere das Verhältnis unter kollegen mit der Vokabel invidia zu charakterisieren pflegt. Das alles setzt aber voraus, daß man in einer Gesellschaft lebte, die bereit war, einem Lehrer der Logik laus et pecunia zu erteilen. Und diese Bereitschaft ist offenbar in einem geradezu verblüffenden Maße vorhanden gewesen.

Ähnliches wie für die Philosophen und Theologen der Francia scheint für die Juristen von Bologna zu gelten. „Zwei Dinge sind's, die die Menschen heftig zur Rechtswissenschaft treiben: Jagd nach Ämtern und eitle Ruhmsucht (ambitio dignitatis et inanis gloria appetitus)." PETER von Blors, der diesen Satz in den 1160er Jahren schrieb, sprach aus Erfahrung; denn er hatte nicht nur in Paris Philosophie, in Tours Rhetorik, sondern auch in Bologna Jurisprudenz studiert, aber dies Studium abgebrochen, um zu den Pariser Theologen zurückzukehren. Seine Karriere aber war die eines Höflings geworden: Prinzenerzieher am Normannenhof in Palermo, dann Sekretär König Heinrichs II. von England und der Königin Eleonore (jener Dame, die nicht nur durch ihre zwei Ehen das aquitanische Erbe zuerst dem König von Frankreich und dann dem von England zubrachte, sondern auch, wie keine andere, die Troubadours in Aufregung versetzte), zuletzt Kanzler des Erzbischofs von Canterbury - das waren die Stationen in Peters Leben, das hier als Beispiel für manche ähnliche Karriere, vor allem im anglo-angevinischen Großreich, genannt sei. Und aus den Kreisen von Bologneser Studenten der Rhetorik und des Rechts um 1120 stammen die ältesten Studentenbriefe, die zum Ausdruck bringen, dab eine bürgerliche Familie vom Studium des Sohnes nicht nur Ehre, sondern auch handgreifliche Vorteile erhofft. Wissensdrang und das Streben nach sozialem Aufstieg verbinden sich, höfische, bürgerliche und gelehrte Kultur durchdringen sich in einer Weise, die zuerst die Schulen füllt und dann zur Bildung der Universitäten beiträgt.

Verlassen wir einen Augenblick die Schulen und sehen auf das höfische Leben der Zeit. Schon um die Mitte des 12. Jahrhunderts finden wir an den größten Höfen die gelehrten Kanzler. Papsi Lucius II. berief 1144 den Engländer RoberTus Puluus, der einst in Oxford Vorlesungen über die Bibel gehalten und später in Paris gelehrt hatte, zum Kardinal und Kanzler der römischen Kirche. 1153 übernahm dies Amt Roland BANDINELLI aus Siena, der einerseits ein theologisches Sentenzenbuch im Sinne der Abaelardschule, anderseits eine Summa zu Gratians Kirchenrechtssammlung geschrieben und in Bologna Theologie mit EinschluB des kanonischen Rechts gelehrl hatte. Aber auch der große politische Antipode Rolands, des späteren Papstes Alexander III., Rainald von Dassel, Kanzler des Kaisers Friedrich Barbarossa seit 1156, war durch die Schulen von Paris gegangen, etwa zur gleichen Zeit wie der dritte der großen Kanzler jener Jahre, Thomas Becket, der dem englischen König Heinrich II. von 1154-1163 diente und dann als Erzbischof von Canterbury des- 
sen großer Gegenspieler wurde. Sie alle finden den Weg von den hohen Schulen zu dem höchsten Hofamt, und nur einer von ihnen, bezeichnenderweise der Deutsche, ist zu solchem $\mathrm{Amt}$ schon durch hochadlige Geburt prädestiniert. Rainalo ist auch gewiß der am wenigsten gelehrte unter ihnen; dennoch verbindet ihn mit den anderen Kanzlern ein bestimmtes weltmännisches Auftreten, ein akademischhöfischer Stil in der Politik.

Zumindest eine Wissenschaft wird an jedem Hofe täglich praktisch gebraucht: die Rechtswissenschaft, insbesondere die Wissenschaft vom kanonischen Recht. Hatten noch unter dem Zisterzienserpapst Eugen $(1145-1153)$ Theologen über die Juristen an der römischen Curie geklagt, deren Spitzfindigkeiten nicht einmal der Papst selbst durchschaue, so wurde dies bald anders; aber nicht weil die Spitzfindigkeiten verschwanden, sondern weil nun der Stuhl Petri selbst von gelehrten Juristen beselzt wurde. Und jeder bischöfliche oder weltliche Hof, der sich mit den römischen Juristen messen wollte, bedurfte seinerseits der gelehrten Kanonisten. Nicht so unmittelbar praktisch anwendbar war die Wissenschaft vom römischen Recht, die Guarnerius um die Wende vom 11. zum 12. Jahrhundert in Bologna belebt hatte. Und dennoch fand auch sie Eingang an den Höfen wie an den Schulen nicht nur in Italien, sondern auch in England und am Kaiserhofe.

Fragt man allein nach der praktischen Bedeutung der Wissenschaften, so wird man ihre Rolle für das höfische Leben wohl nicht voll ermessen können. Die Ubersetzer am Hofe der normannischen Könige, die arabische und griechische Philosophie der lateinischen Welt zugänglich machten, Bischof HeINRICH VON WINChester, der römische Antiquiläten sammelte, der Erzpoet, der geistvolle Vaganten-Verse für RAINALD vON DASSEL schmiedete, der kaiserliche Pfalzdiakon Hugo von Honau, dessen theologische und philosophische Traktate eben erst im Druck erschienen sind - sie alle legen, jeder in seiner Weise, Zeugnis dafür ab, daB das höfische Leben geistreiche Männer anzog, daB WVitz, Esprit und Courtoisie ebenso wie echte Wissenschaft und Weisheit ihre Gönner an den geistlichen und weltlichen Höfen fanden. Die allgemein bekannte höfische Dichtung in den Volkssprachen gehört ebenso dazu wie die Werke der Gelehrten und die Verse vieler Vaganten. Der Hof bedarf der literati nicht nur als Fachleute, sie sind notwendiger Bestandteil seiner Selbstverwirklichung. Dic neue Vokabel curialitas meint jene weltgewandte Bildung, die den curialis, den Höfling, auszeichnet.

Einklang und Spannung zwischen gelehrter und höfischer Welt zeigt uns am deutlichsten .Johannes von Salisbury. Zwölf Jahre hatte er in Paris und Charlres studiert, zwölf weitere Jahre in England und in Rom das Leben eines Höflings geführt, als er $1159 \mathrm{dem}$ Kanzler Thomas Becket sein großes Werk Policraticus sive de nugis curialium et vestigiis philosophorum widmete. Mit Ironie und scharfer Kritik verfolgt er die höfischen Vergnügungen, Jagd und Würfelspiel, Zauberei und Wahrsagerei, Traumdeutung und was dergleichen geistreich-höfischen Vergnügens mehr ist. Er hat es bis zum Uber- 
druß und Ekel kennengelernt und setzt nun seine christlich-humanistisch begründete Sozial- und Fürstenethik, eine der bedeutendsten des Mittelalters überhaupl, dem entgegen. Dabei zielt seine Kritik nicht auf die ritterlichen illiterati, sondern gerade auf die Gebildeten unter den Höflingen, die literati, die die Spuren der Philosophie verlassen haben, um ihren nugae zu folgen. Aber Johans will nicht höfische und gelehrte Welt trennen, sondern vielmehr dem höfischen Leben eine tiefere geistige Basis geben. Der Anspruch geht noch weiter: auch Johann von Salisbury zitiert das jetzt öfter genannte Wort: „Rex illiteratus quasi asinus coronatus." Auch der König soll literatus sein - und Heinrich II. von England ist es in gewisser Weise wirklich.

Die literati waren aber nicht nur ein fester Bestandteil des höfischen Lebens; auch die Städte bedurften ihrer jetzt. Ihr Anteil, insbesondere der Anteil der Juristen, am Werden der europäischen Stadtkultur und am Aufstieg der städtischen Freiheit ist noch sehr wenig erforscht; aber mit Recht hat man darauf hingewiesen, daß insbesondere in Italien und Südfrankreich - anders als an der Maas und am Rhein - die Kommunen schon im 12. Jahrhundert ganz wesentlich von den Juristen mitgetragen wurden. Wenn Friedrich Barbarossa zum ronkalischen Reichstag von 1158, der die Kaiserrechte in den Städten definieren sollte, die berühmten vier Bologneser Doktoren heranzog, so waren diese nicht nur Experten des alten, justinianischen Kaiserrechtes, sondern auch von den Bürgern anerkannte Rechtsautoritäten. In der Bologneser Quästionenliteratur werden schon damals Fragen des städtischen Statutenrechtes mit Hilfe romanistischer Quellen und Methoden gelöst. Unentbehrlich für den immer komplizierter werdenden Handel, insbesondere mit dem Orient, wurden die Notare, die in den Städten einen bedeutenden Stand ausmachten und durch ihre Dienste für das tägliche Leben der Handelsherren, die vor ihnen Handels- und Eheverträge abschlossen, zu Ansehen und Geld gelangten; und diese Nolare hatten in stets wachsendem Maße in Bologna studiert oder zumindest durch die Schriften der Bologneser Meister gelernt. Denn schon Guannerius hat neben gelehrten Glossen zu den Digesten auch praktische Lehrbücher und Formulare für Notare verfaßt, und die Doktoren der nächsten Generationen folgten darin seinem Beispiel. War das römische Recht auch nirgends schlechthin geltendes Recht, so gab es doch seinen Adepten eine Methode an die Hand, die ihnen Überlegenheit und Autorität in allen verwickelten Fragen verlieh, die das bürgerliche Leben mit den überlieferten Normen nicht mehr zu lösen vermochte. So wächst, zunächst in Italien, ein literarisch gebildeter Laienstand. Der Genueser Konsul Cafaro, der Pfalzrichter OTto Morena aus Lodi und der anonyme MaILÄnder AnNalist sind seit Jahrhunderten die ersten Verfasser großer historischer Werke, die aus dem Laienstand kommen; und sie alle bekleiden Stellen von hohem Rang in ihren Kommunen. An wissenschaftlicher Bedeutung wurden sie übertroffen durch die drei Pisaner Hugo Etherianus, L.eo Tuscus und Bukgundio, die alle große theologische und philo- 
sophische Werke aus dem Griechischen ins Lateinische übertrugen und zum Teil selbst verfaßten. Unter ihnen haben HUGO und LEO lange im Dienst des Kaisers Manuel von Byzanz gestanden, zugleich aber auch politisch für ihre Heimatstadt und deren Kolonie in Konstantinopel gewirkt, während Burgundo als Richter und Konsul seiner Vaterstadt hervortrat, durch die Dedikation einer seiner Übersetzungen an Friedrich Barbarossa aber auch den Bogen zu den höfischen Kreisen spannte. Uberschaut man die Lebensläufe und die erst jüngst veröffentlichte Korrespondenz dieses Kreises, so sieht man, wie Konstantinopel und Pisa, die Höfe der Babenberger und der Staufer, die Schulen von Bologna und Paris durch ein Netz vielfältiger Beziehungen miteinander verknüpft werden. Und von Generation zu Generation steigt das Ansehen und die praktische Bedeulung der Juristen in den Städten. Schon um 1240 wirken selbst in norddeutschen Städten gelehrle Ratsschreiber, und es ist methodisch richtig, wenn man sich fragt, ob sie in Bologna oder Paris studierl haben - ein dritles kommt kaum in Frage.

Nicht zuletzt werden kirchliche Würden und Pfründen in zunehmendem Maße den gelehrten oder doch zumindest akademisch ausgebildeten Bewerbern zuteil, auch wenn diese weder dem Adel entstammen noch spezielle kirchliche oder asketische Verdienste geltend machen können. Die um 1150 in Paris lehrenden Magister erreichen fast alle Bischofsstühle: Gilbert Ponneta in Poitiers, der Lombarde Petrus in Paris selbst, Robert von Melun in Hereford (England), ADAM VON DER KLEINEN BRÜCKE in St. Asaph (Wales); freilich war auch der Hof des Erzbischofs Theobald von Canterbury ein Bildungszentrum von solchem Niveau, daß er hochgelehrte Bischöfe liefern konnte, die Frankreich nie besucht halten. Aber im anglo-französischen Kulturkreis kann man jetzt fast nur noch auf Grund hoher Bildung Bischof werden. Auch aus Deutschland zieht mancher Adlige in die Irancia - OTto von Freising ist der bekannteste -, aber noch um 1140 vermerkt man es als unerhört rühmenswert, daß ein Saarbrücker Grafensohn und Neffe des Erzbischof's von Mainz, der doch allein auf Grund seiner Herkunft leicht Karriere machen komnte. sich doch den Strapazen eines Studiums in Reims unterzieht. Hier bleiben die Bistümer dem Adel vorbchalten, ob gebildet oder nicht, aber manch andere Pfründe öfnet sich dem Bürgerlichen durch das Studium. Keine Diözese kann mehr ohne akademisch gebildete Geistliche verwaltet werden.

Wir haben gesehen, daß an den königlichen und bischöflichen Höfen ebenso wie in den Städten im Laufe des 12. Jahrhunderts eine Art neter Stand - noch kein abgeschlossener Rechtsstand, aber ein Berufssland - auftritt. Das spiegelt sich auch in dem Aufkommen neuer Bedeutungen für alte Standesbezeichnungen. Die alte, durch Tonsur und Weihen bestimmte Scheidung zwischen Klerikern und Laien vermischt sich, wenn immer mehr Scholaren zwar die Tonsui nehmen und damit rechtlich Kleriker werden, aber die sakramentalen Weihen nicht folgen lassen. Schon un 1115 bemerkt der Mönch RLPERT vON DEUTz mit Mißfallen, es sei neuerdings üblich geworden. 
einen jeden sehr gebildeten Mann, welches Standes oder habitus er auch sei, als clericus zu benennen. Die Bildung macht den Stand aus. Selbst die Päpste unterscheiden im 13. Jahrhundert zwischen Scholaren und Laien. Damit setzt eine Entwicklung ein, die schlieBlich dazu führt, da $B$ der laicus identisch mit dem illiteratus ist, während der Schreiber im Französischen le clerc heißt. In Sizilien zitiert das Geselzeswerk Rogers II. 1140 das Wort, die Juristen seien Priester des Rechtes, und um die gleiche Zeit beginnt man in der Lombardei diejenigen Richter, die nicht iuris periti sind, $d$. h. keine juristische Ausbildung haben, als laici zu bezeichnen. Das Recht geht aus der Hand der durch Geburtsstand und Uberlieferung als weise geltenden Männer in die der Fachleute über, und im 13. Jahrundert behalten städtische Statuten es diesen ausdrücklich vor - der Unausgebildete, der laicus, wird vom Richteramt ausgeschlossen. Das ständische Selbstbewußtsein der Bologneser Scholaren findet schon um 1170 seinen Ausdruck darin, daß diese nicht nur als "Herren“ — domini oder signori - angeredet sein wollen, sondern auch ritterliche Standesrechte beanspruchen. Der akademisch Gebildete tritt in den Wettbewerb mit dem durch Geburtsstand hervorgehobenen Richter. Einzelbeispiele dafür, etwa anläßlich der Vergebung königlicher oder kirchlicher Ämter, ließen sich in Menge anführen - bis hin zu jenen lateinischen Streitgedichten, in denen die Mädchen darum zanken, ob der Ritter oder der Clericus besser zur Liebe geeignet sei, und aus diesem Anlaß die Vorzüge und Nachteile beider Stände ausführlich schildern.

Zur Geschichte der hohen Schulen und Universitäten gehört als notwendiges liorrelat die Sozialgeschichte der akademisch Gebildeten, und hier slehen wir noch ganz am Anfang der Forschung. Das gill auch für die Entstehungszeit der ersten Universitäten. Während man die Zeugnisse über diese selbst, ihre Institutionen und ihre Lehrer verhältnismäßig sorgfältig gesammelt hat, bedarf es noch mühsamer Kleinarbeit, um die weit verstreuten Zeugnisse über Leben und Wirken derer zu sammeln, die die hohen Schulen in deren erster Zeit besucht haben, um dann an den Iö̈fen und in den Städten zu wirken. Erst wenn dies geschehen ist, wird man genauer verfolgen können, was ich hier an einigen markanten und bekannten Beispielen zu belegen versuchte, die Verflechtung der akademischen Gesellschafi mit der höfïschen und der bürgerlichen.

Auch Rittertum und Bürgertum sind eben erst im 11. und 12. Jahrhundert dahin gelangt, eigene Stände zu bilden. Beide überbrücken mannigfache alte Unterschiede rechtlich freier und unfreier Abkunft durch soziale und berufliche Gemeinsamkeiten, und sie gelangen durch genossenschaftliche linung zur Bildung neuer sozialer Formen. Und auf solcher Einung beruht auch der Schritt von der Vielzahl unsteter Schulen im frühen zu den wenigen Universitäten im späten 12. Jahrhundert.

Das wesentlich Neue an den Schulen im frühen 12. Jahrhundert war die Freizügigkeit, sowohl der Lehrer als auch der Scholaren, gewesen. Aber in einer Zeit, da es kein allgemeines Bürgerrecht gab, 
sondern ein jeder in den Rechtskreis gebunden war, dem er durch Stand und Geburt zugehörte, da jeder nur dort Recht finden konnte, bedurfte die Freizügigkeit des Schutzes, der in doppelter Weise begründet werden konnte: von den Scholaren und Magistern selbst durch genossenschaftliche Einung, die einen neuen, eigenen Rechtskreis schuf, und von den geistlichen und welllichen Obrigkeiten durch Anerkennung und Privilegierung dieses Rechtskreises. Schon in den $1130 \mathrm{er}$ Jahren finden wir die Reimser Studenten in landsmannschaftlichen Gruppen beisammen; neben Franzosen und Engländern bilden die Deutschen eine eigene - schon damals durch ihre sonderbaren Sauf- und Raufsitten bekannte - Gruppe: in ihrer Gesamtheit sind die Scholaren bei den üblichen Schlägereien den Bürgern überlegen. 1158 erläBt Friedrich Barbarossa, beraten von den Bologneser Doktoren, das erste Scholarenprivileg, nicht für eine abgegrenzte Gruppe, sondern als ein dem Codex Justinians einzufügendes allgemeines Gesetz, die berühmte Authentica „Habila“. Sie verbietet insbesondere, Scholaren in der Fremde für die Schulden ihrer Landsleute haftbar zu machen, und gesteht den Studenten den Gerichtsstand unter ihrem Lehrer oder dem Bischof der Hochschulstadt zu. Rechtsschutz in der Fremde, das ist das erste, dessen die Scholaren bedürfen, und so ist denn die Bologneser Studentenuniversität aus den zunächst vier, dann zwei landsmannschaftlich unterschiedenen universitates, $d . h$. Genossenschaften, der Fremden in Bologna hervorgegangen; weder die in Bologna beheimateten Studenten noch die dort Bürgerrecht genießenden Doktoren hatten einen Anlaß, Glieder einer solchen Genossenschaft zu werden.

In Paris schlossen sich zunächst die Magister zusammen; Reibungen mit dem Kanzler mögen dabei mitgewirkt haben - in jedem Fall war die invidia der Abaelardzeit auf die Dauer keine Grundlage für die Existenz vieler Lehrer an einem Ort; gemeinsame Interessen zwangen sie zur rechtlichen Einung. Dem Kanzler des Bischofs stand das Recht $z u$, die licentia docendi zu erteilen, aber erst die Aufnahme in die Genossenschaft der Magister ergab die Möglichkeit, von dieser Lizenz Gebrauch zu machen. Der Abschluß einer Gemeinschaft nach außen und die Einführung feierlicher Aufnahmeriten korrespondieren miteinander; die öf fentliche inceptio - eine Art Antrittsvorlesung oder Antrittsdisputation - ist geradezu als der Ursprung der universitas selbst bezeichnet worden. Sie hat ihre Parallelen in Ritterweihe und Ritterschlag, in Bürgereid und feierlicher Aufnahme unter die Meister einer Zunft.

Warum aber erreichte dies Ziel des genossenschaftlichen Zusammenschlusses, das Ziel der universitas, nur Paris - nicht Chartres. Reims oder Laon? Es ist, wie mir scheint, unverkennbar, daß seit den späten Jahren Peter Abaelards die Schulen von Paris eine ständig wachsende Zahl von Magistern aufweisen. Um 1140 geht selbst der Kanzler von Chartres, der große Logiker und Theologe GiLbert, nach Paris. Keine Quelle nennt uns Zahlen, keine Quelle nennt uns die Gründe; aber es will scheinen, daß es zunächst die Studenten sind, die von Paris angezogen werden, und daß die Lehrer ihnen fol- 
gen, weil sie dort mehr Möglichkeiten des Verdienstes finden. Als Johann von Salisbury 1164 auf der Flucht vor seinem König nach Paris zurückkommt, da bricht er in Entzücken über die Stadt seiner einstigen Studien aus. Die Fülle an Lebensmitteln, die Fröhlichkeit des Volkes, die Würde des Klerus, der Ruhm und die Geschäftigkeit der Gelehrten veranlassen ihn, die Stadt mit Jakobs Himmelsleiter zu vergleichen, auf der die Engel auf- und niedersteigen, und mit den Worten des alttestamentlichen Patriarchen ruft er aus: „Wahrlich, der Herr ist an diesem Orte, und ich wußte es nicht!" Und er zitiert das Dichterwort: „Felix exilium, cui locus iste datur". Aber sein Freund Peter von Celle warnt ihn: „O Paris, wie bist du fähig, die Seelen zu fangen und zu betrügen. Du hast Netze des Lasters, Fallstricke des Bösen, in dir trifft der Höllenpfeil die Herzen der Toren": und für JoHAnNes selbst kam die Ernüchterung rasch: nach langer Suche erst fand er ein Quartier, das er erst beziehen durfte, nachdem er 12 Pfund für die Miete eines ganzen Jahres im voraus erlegt hatte - nicht nur seine ganze Barschaft, auch seine Pferde hatte der Verbannte opfern müssen.

Wer Weisheit und Wissenschaft nicht in der Einsamkeit eines Klosters, sondern in der Welt suchte, dem bot Paris mehr an Welt als Chartres, Laon oder Reims. Eben in der Zeit König Ludwigs VII. wurde Paris immer mehr zur Königsstadt, immer mehr zum Sitz des Hofes und zur großen Stadt. Hier gab es mehr Verbindungen zu höfischen und bürgerlichen Kreisen, mit einem Worte: wer mit den Studien auch die große Welt suchte, der fand hier alles, vom König bis zum Freudenmädchen. Zugleich mußte aber auch die Vielzahl der mit- und gegeneinander lehrenden Magister ihren eigenen Reiz auf die ausüben, denen es um die Wissenschaft selbst ging.

So wird Paris zunächst zur rein zahlenmäßig den anderen überlegenen hohen Schule, sodann aber auch zur geistigen Autorität und die Genossenschaft der Lehrenden gewinnt damit diejenige äuBere Macht, die zur Begründung und Anerkennung der neuen sozialen Form notwendig ist. Der blutige Konflikt zwischen der universitas einerseits, den Bürgern und dem königlichen prévôt anderseits, den eine Wirtshausschlägerei im Jahre 1200 hervorgerufen hatte, endete mit dem vollen Sieg der Universität durch die Intervention König Philipps II. August und mit der Erteilung des ersten königlichen Privilegs für die Universität. Dabei hatte bereits die Drohung der Magister, Paris zu verlassen und die Universität an einen anderen Ort zu verlegen, mitgewirkt - eine Drohung, unter der die Universitäten der folgenden Jahrhunderte immer wieder standen und die, hier und dort wahrgemacht, zu manchen Neugründungen führte. Man war stark genug, drohen zu können; denn die Universität besa $B$ zugleich geistige Autorität und bedeutete mit ihren im 13. Jahrhundert wohl 6000-8000 Studenten in Paris einen wirtschaftlichen Faktor, mit dem nicht nur Städte, sondern auch Könige rechnen mußten.

Freilich, die Konzentration der theologisch-philosophischen Wissenschaften auf Paris bedeutet nicht nur Gewinn und Vertiefung, 
sondern auch Verlust. Schon in der zweiten Hälfte des 12. Jahrhunderts gehen die Schulen von Chartres, Reims u. a. merklich zurück, und das heißt nicht nur, daß Bildungsstätten verloren gehen. In Paris ist allein das scholastische Distinguieren und die Disputatio die weiterführende Methode - der Humanismus von Chartres, die Lektüre der Auctores und die Bildung des Menschen an ihnen, wie sie einem JoHann von Salisbury als Ziel vorgeschwebt hatte, hat keine Zukunft mehr. Zugleich vermögen die kỉöster nicht mehr jene schöpferische Theologie fortzuführen, die Männer wie Hugo von ST. Vikton, Bernihard von Clairvaux und Rupert von Deutz gepflegt hatten und die das Bild des 12. Jahrhunderts so reich macht. Diese klösterliche Theologie ist keineswegs so einseitig konservativ, wie die neuere Scholastik-Forschung oft gemeint hat; sie hat in RupERTs Biblizismus und in Bernhards Mystik, im Symbolismus HILdegards voN BINGEN und anderer tiefe und neue Ideen, die von der Scholastik der Universität beiseite gedrängt werden.

In Streit mit den Theologen von Laon war Rupert der Neuerer, der Augustins Autorität relativieren und nur die biblische Autoritäl als absolut anerkennen wollte, der den Anspruch erhob, proprii ingenii vomere den Acker der Heiligen Schrift zu bestellen; und wenn auch Bernhard die großen Angriffe gegen AbaElari) und GILbERT vortrug, so finden wir doch gerade unter Zisterziensern und Augustinerchorherren eifrige Anhänger dieser verketzerten Meister. Aber die scholastische Wissenschaft lebte, wie ihr Name sagt, von der Schule, und diese fand ihre gültige Form nicht mehr im kloster, sondern in der neuen universitas. Selbst die Schule des Klosters Sankt Viktor in Paris kann nicht länger als zwei, drei Generationen während des 12. Jahrhunderts fruchtbar bleiben. Das Benediktinertum und das Regularkanonikertum mit ihrer Bindung an die stabilitas loci vermögen nicht, sich selbst in die neue soziale Gestalt der Schule, in die universitas, hineinzubegeben, und damit verkümmern ihre Schulen und ihre geistigen Traditionen, nachdem erst einmal die Universität sich durchgesetzt hat und die geistig regsamen Kräfte anzieht. Erst das Bettelmönchtum mit seiner ganz anderen Ordensverfassung ist auf das Wirken in den Städten und im Volke eingestellt und verschafft sich - gegen den hartnäckigen Widerstand der Universität - Eingang in die Universität selbst und damit einen neuen Wirkungskreis des Mönchtums, ohne doch die im 12. Jahrhundert abgebrochenen geistigen Traditionen neu beleben zu können.

Versuchen wir unsere Skizze zusammenzufassen. Geistige Impulse lassen seit der Wende vom 11. zum 12. Jahrhundert Schulen aufblühen, die in Auseinandersetzung mit der kirchlichen und welllichen Gesellschaft schlieblich um 1200 ihre gültige Gestalt in der neuen Universität finden. Untrennbar verbunden ist dieser Prozeß mit dem Entstehen eines akademischen Standes, der an den Höfen, in der Kirche und in den Städten wirkt und durch akademische Grade Magister, Doktor - eine allgemein und allerorten anerkannte, dem Ritterlum und der Meisterschaft im Handwerk vergleichbare Würdo erhält. Das Werden der Universität und des akademischen Standes 
stehen in einer Wechselbeziehung, die verbietet, das eine als Folge des anderen zu deuten. Die Schulen des 12. wie die ausgebildete Universität des 13. Jahrhunderts haben sich nie das Ziel gesetzt, die Höfe und Städte mit Fachleuten zu versorgen. Wohl aber ist die soziale Geslalt der Universität durch die Gesellschaft mitgeformt worden; ja, erst das lebhafte Interesse weiter Kreise hat den hohen Schulen die Möglichkeit gegeben, zu beständigen und unabhängigen Einrichtungen zu werden. Von Anfang an steht die Lehre in der Spannung zwischen dem ursprünglichen Trieb, die Wahrheil zu suchen, und dem Wunsch vieler, praktische Ausbildung zu finden. Umgekehrt formen die Schulen, ohne dies eigentlich zu wollen, den neuen akademischen Stand und verändern das gesamte Gefüge der Gesellschaft wesentlich, machen es reicher und komplizierter.

Staatliche und kirchliche Autorität sind am Entstehen der Universität zunächst allenfalls mittelbar beteiligt; erst nachträglich sind Landesherren, wie Kaiser Friedrich II. in Neapel, auf die später oft imitierte Idee gekommen, Staatsanstalten zur Erziehung der Landeskinder und zur Versorgung des Reiches mit Beamten zu schaffen, also Einrichtungen, die weder studium generale noch universitas im echten Sinne waren. Die Auseinandersetzung mit staatlicher und kirchlicher Autorität bildet seit dem 13. Jahrhundert ein wichtiges Kapitel der Universitätsgeschichte, das nicht nur von mancher Unterdrückung zu reden weiß, sondern auch von jener Verführung, da die Pariser Universität auf dem Weg über die Konzilien des 15. Jahrhunderts die Entscheidungen der großen Politik von Staat und Kirche an sich zu ziehen suchte - und dabei mit dem politischen Ansehen auch ihre geistige Freiheit verlor. Die Aufgabe, zwischen dem Anspruch der Gesellschaft und der politischen Autoritäten einerseits und dem geistigen Imperativ anderseits ihren eigenen Weg zu finden, ist der Universität gestellt, solange sie noch einen Rest der Unabhängigkeit und der genossenschaftlichen Verfassung ihrer Ursprünge zu bewahren vermag. 